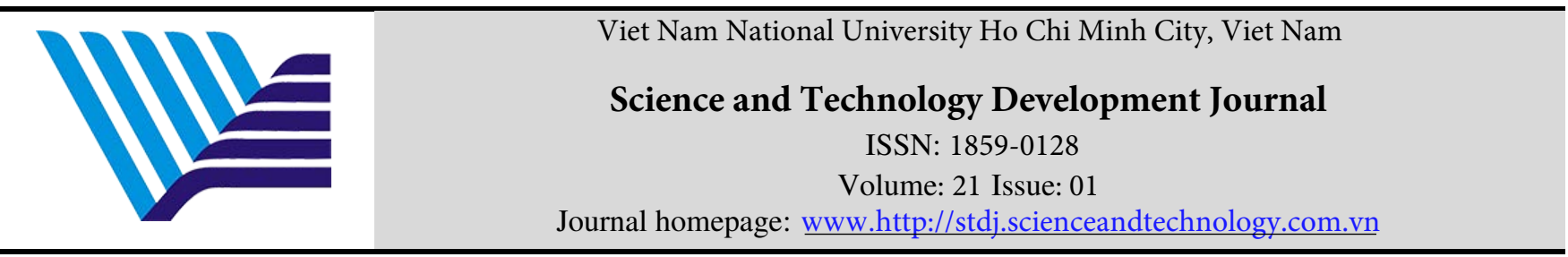

\title{
Dynamic analysis of knee-exoskeleton and its implementation in design of magnetorheological damper
}

\author{
Bui Quoc Long Mai, Van Chi Le, Tran Huy Thang Le, Xuan Phu Do* \\ MediRobotics Laboratory, Department of Mechatronics and Sensor Systems Technology, Vietnamese \\ German University, Binh Duong, Viet Nam
}

\begin{tabular}{l} 
ARTICLE DETAILS \\
History \\
Received: 04 April 2018 \\
Accepted: 12 May 2018 \\
Published: 02 June 2018 \\
\hline
\end{tabular}

Keywords

ANSYS ADPL, Force analysis,

Knee exoskeleton, Moment

analysis, MR damper,

Optimization

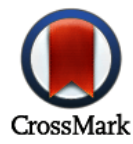

\begin{abstract}
In this study, a new development of knee exoskeleton is presented. This structure helps people who have difficulties in walking, especially those whose knee functions are broken or weak. The design includes 3 main components: motor, damper, and mechanical frame. The user movement is controlled by using a magnetorheological (MR in short) fluid in the damper. The operation of the damper is based on the change of viscosity of MR fluid to variations in the magnetic environment. To design the damper, kinematic parameters of the proposed exoskeleton were analyzed to find relations among forces and torques. After analysis, the function of the damping force was established. The magnetic flux density and yield stress functions were also found; these functions are the basis for derivation of the damping force formulation. The forces from the exoskeleton are directly related to the damping force. Hence, these functions are used as an objective function to find the optimized geometry of the damper. The optimization process was done using ANSYS ADPL. Our study showed that the requirements for the design of the damper can be obtained and used in manufacturing in the future.
\end{abstract}

(C) 2018 Viet Nam National University Ho Chi Minh City, Viet Nam under a Creative Commons Attribution-NonCommercial-ShareAlike 4.0

\section{Introduction}

Nowadays, the application of MR actuator in industry is popular, such as in automobile suspensions, household appliances, and medical devices. This application demonstrates the importance of the role of MR fluid in controlling vibration and the complicated environment that conventional devices cannot

${ }^{\star}$ Corresponding author's email address: phu.dx@ @gu.edu.vn 
solve or control. A new bypass valve for MR damper was proposed in McLaughlin et al. (2014). This configuration was a spiral rod to adjust the flow of MR fluid. The operation of this valve was similar to the conventional valve, but it focused on improving the flow of MR fluid. A review of the configurations for designing a high loaded MR mount used for vibration control was presented in Do and Choi (2015), where a combination of flow and shear modes was exploited. Similar principles of magnetorheological fluid, such as flow mode, shear mode, and squeeze mode in Do and Choi (2015), can be used for both damper, mount, and brake. In addition, simulation of MR actuator was also presented in Do and Choi (2015), where the relation between the load and inside pressure was clearly analyzed.

In the design of the MR actuator, the parametric model is the first requirement to building the structure. The expression of all variables of the parametric model must be presented clearly. The problem of the parametric model was studied in et al. (2010), which showed that simple algebraic parametric models could more accurately capture the non-linear hysteristic behavior of MR damper compared to the differential parametric models. The dynamic analysis of the duct flow model for design of the damper was also studied in Esteki et al. (2014). The duct flow model could be used for electrorheological (ER) fluid, which has a larger energy for control than MR fluid. However, it should be remarked that the duct flow is restricted for design of the MR actuator because the efficient gap must in the range of 1-2 mm. The vibration control of the MR damper system were studied in Do et al. (2015); Choi et al. (2016); Phu et al. (2017c,b,a). In these studies, the control of the MR damper could combine with both conventional controls (such as optimal control, PID control, etc.) and modern controls (such as fuzzy model, neural networks model, etc.). The key of these combinations is from the varied energy of the MR damper following the change of applied current or magnetic field. Hence, the feedback signal of control and the calculated signal of the algorithm can be used to change the energy of the damper.

Another configuration following the flexible gap of MR mount was studied in Phu et al. (2014a), where the changeable damping gap showed a superior performance. This design was used for the MR actuator operating under multi-dimensional load. In addition, this design requires a high precision of manufacturing to improve the ability of self-positioning. The combination of MR brake and mount was studied in Phu et al. (2014b). This idea is based on a damper design which can control both vertical and horizontal vibrations. The combination of damper and brake is useful of controlling vibrations of ship engines, where the loads always exist in two directions. The design of the damper, and annular and radial fluid flow were presented in Wang et al. (2009). This structure is used for high-loaded MR actuators because the high pressure during the operation can suffer from high loads; also, the structure controls these loads better than the conventional structure.

From these studies, the configuration of the damper is focused on the design of annular and radial flow of MR fluid. The efficiency of this configuration has been proven with many commercial MR actuators. Hence, this configuration is the first choice in the design of the new MR actuator for special applications.

In this study, the new configuration of exoskeleton with two MR dampers was proposed. The calculations of force and moment were first carried out; then, the configuration of the MR damper was proposed and optimized, following the initial requirements.

\section{Configuration of New Proposed Exoskeleton}

\subsection{Configuration}

Configuration of the proposed exoskeleton is shown in Figure 1. The exsoskeleton includes three parts as follows: one motor supporting the torque (for the user to pull his leg up), the first damper (used for both reducing vibrations during motion, and making auto-motions more soft and comfortable), and the second 
damper (combined with a spring for reducing vibrations when the leg lands on the ground, and supporting force for the user).

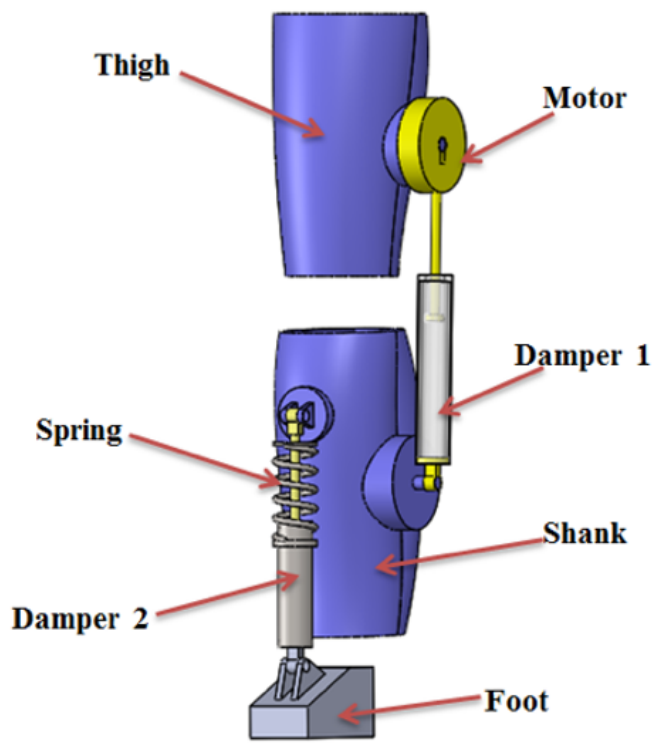

Figure 1. Exoskeleton configuration.

\subsection{Force and Torque Calculation of the proposed exoskeleton}

The mechanical framework of the exoskeleton is shown in Figure 2. To make it simpler and easier during the calculation process, the thigh, shank, dampers and foot are bars, and are approximated. Thus, the mass center of each section is at the midpoint.

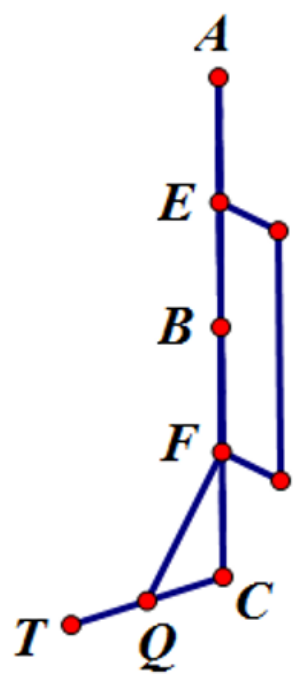

Figure 2. Mechanical model of the proposed exoskeleton.

In this calculation, the change of the damper lengths when the leg is in motion was evaluated. Firstly, the geometric parameters of an individual when pulling the leg up at angle was shown in Figure 3. The 
Table 1. Kinematicand dynamic parameters

\begin{tabular}{|c|c|c|c|}
\hline $\begin{array}{l}\text { Sym- } \\
\text { bol }\end{array}$ & Definition & $\begin{array}{l}\text { Sym- } \\
\text { bol }\end{array}$ & Definition \\
\hline $\mathbf{A}$ & Upper joint & $l_{6}$ & $C F$ \\
\hline $\mathbf{B}$ & Knee & $\varphi$ & $J A K$ \\
\hline $\mathbf{C}$ & Lower joint & $\varphi_{2}$ & $\pi / 2-\varphi$ \\
\hline $\mathbf{E}$ & Motor & $m_{1}$ & Mass of shank \\
\hline $\mathbf{F}$ & Tip of damper (1) & $m_{2}$ & Mass of thigh \\
\hline $\mathbf{Q}$ & Tip of damper (2) & $m_{3}$ & Mass of motor \\
\hline $\mathbf{K}$ & Mass center of damper 1 & $m_{4}$ & Mass of damper (1) \\
\hline $\mathbf{K}_{2}$ & Mass center of damper 2 & $m_{5}$ & $\begin{array}{l}\text { Mass of damper ( } 2 \text { ) } \\
\text { (including mass of spring) }\end{array}$ \\
\hline$l_{1}$ & $\mathrm{BF}$ & $m_{6}$ & Mass of foot \\
\hline$l_{2}$ & $\mathrm{BE}$ & $M_{E}$ & Motor torque \\
\hline$l_{3}$ & Damper length function (1) & $M_{A}$ & Upper human torque \\
\hline$\alpha$ & 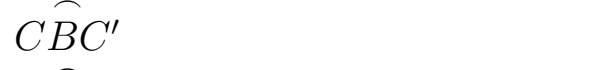 & $M_{B}$ & Knee Torque \\
\hline$\alpha_{2}$ & $B \widetilde{E} F^{\prime}$ & $M_{C}$ & Ankle torque \\
\hline$\beta$ & $\pi-\alpha$ & $P_{i}$ & $\begin{array}{l}m_{i} g \\
(i=1,2,3,4,5,6)\end{array}$ \\
\hline$\alpha_{3}$ & $F^{\prime} C F$ & $\theta$ & $\pi-\alpha_{2}-\varphi$ \\
\hline$\alpha_{4}$ & $Q \widehat{F^{\prime} C}$ & $\gamma$ & $\pi-\alpha-\varphi$ \\
\hline$l_{4}$ & Damper length function (2) & $\alpha_{5}$ & $\alpha_{4}+\pi-\alpha 2-\varphi$ \\
\hline$l_{5}$ & QC & $\alpha_{6}$ & $\alpha+\varphi-\alpha_{3}-\pi / 2$ \\
\hline$F_{\text {damper } 1 x}$ & $\begin{array}{l}\text { Horizontal component of damping } \\
\text { force (1) }\end{array}$ & $F_{\text {damper } 2 x}$ & $\begin{array}{l}\text { Horizontal component of damping force (2) } \\
\text { and spring }\end{array}$ \\
\hline$F_{\text {damper } 1 y}$ & $\begin{array}{l}\text { Vertical component of damping } \\
\text { force }(1)\end{array}$ & $F_{\text {damper } 2 y}$ & $\begin{array}{l}\text { Vertical component of damping force }(2) \\
\text { and spring }\end{array}$ \\
\hline
\end{tabular}

results of the length of the first damper and angle $\alpha_{2}$ are shown in equations (1) and (2). The rotation of the ankle within an angle was calculated as shown in Figure 3. The length of the second damper and $\alpha_{4}$ were obtained in equations (3) and (4), as follows:

$$
\begin{aligned}
& l_{3}(\alpha)=\sqrt{l_{1}^{2}+l_{2}^{2} 2 l_{1} l_{2} \cos (\pi-\alpha)} \\
& l_{3}\left(\alpha_{3}\right)=\sqrt{l_{5}^{2}+l_{6}^{2}-2 l_{5} l_{6} \cos \left(\frac{\pi}{2}-\alpha_{3}\right)} \\
& \alpha_{2}=\arccos \left(\frac{l_{2}^{2}+l_{3}^{2}-l_{1}^{2}}{2 l_{1} l_{3}}\right)
\end{aligned}
$$




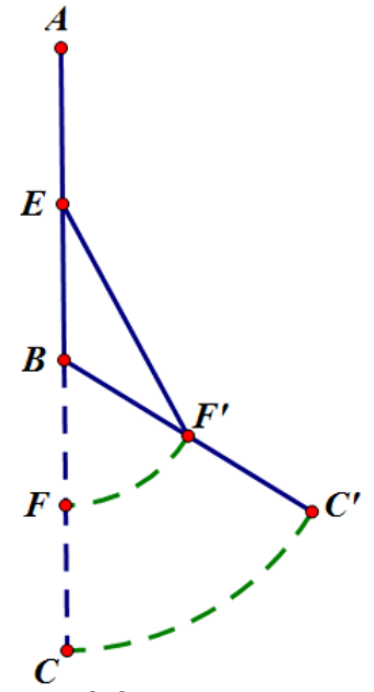

(a)

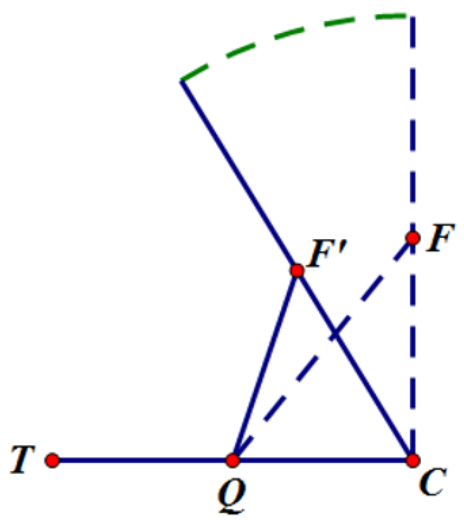

(b)

Figure 3. (a) Geometry of pulling the leg up at angle $\alpha$, (b) Geometry of rotation of the ankle within an angle $\alpha_{3}$.

$$
\alpha_{4}=\operatorname{arcos}\left(\frac{l_{4}^{1}+l_{6}^{2}-l_{5}^{2}}{2 l_{4} l_{6}}\right)
$$

The kinematic relation of motions was continuously analyzed. In this analysis, an assumption was used that the walk of humans is velocity $x(\mathrm{~m} / \mathrm{s})$ with no acceleration. The length of step is $y(\mathrm{~m})$, and the time to accomplish one step is $x / y$ (s). For more ease of calculation as well as design, the position of the motor is at the mass center of the thigh, and the damper tips are at the mass center of the shank and foot. Generally, a person pulls his leg up, the kinematics of motions are obtained, as shown in Figure 4.

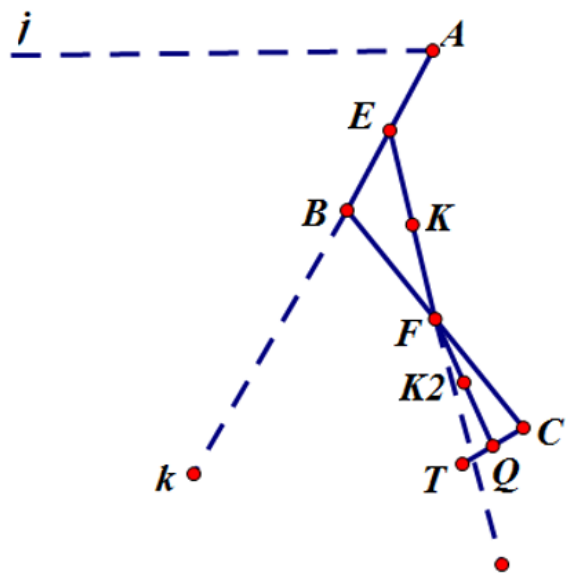

Figure 4. Kinematicgeometry when a person pulls his leg up.

Based on polar coordinates, the kinematics parameters of important points were derived as below:

\section{Point A:}




\section{Point B:}

$\overrightarrow{a_{B}}=2 l_{2} \ddot{\varphi}_{2}\left(\begin{array}{c}\sin \varphi \\ -\cos \varphi\end{array}\right)+2 l_{2}\left(\begin{array}{c}\cdot \\ \varphi_{2}\end{array}\right)^{2}\left(\begin{array}{c}\cos \varphi \\ \sin \varphi\end{array}\right) ; \overrightarrow{v_{B / A}}=2 l_{2} \dot{\varphi}_{2} \overrightarrow{e_{\varphi 1}} ; \overrightarrow{r_{B / A}}=2 l_{2} \overrightarrow{e_{r 1}}$

\section{Point E:}

$\overrightarrow{a_{E}}=l_{2} \ddot{\varphi}_{2}\left(\begin{array}{c}\sin \varphi \\ -\cos \varphi\end{array}\right)+l_{2}\left(\begin{array}{c}\cdot \\ \varphi_{2}\end{array}\right) 2\left(\begin{array}{c}\cos \varphi \\ \sin \varphi\end{array}\right) ; \overrightarrow{v_{E / A}}=l_{2} \dot{\varphi} \vec{e}_{\varphi 1} ; \vec{r}_{E / A}=l_{2} \vec{e}_{r 1}$

\section{Point C:}

$\vec{a}_{C}=2 l_{2} \ddot{\varphi}_{2}\left(\begin{array}{c}\sin \varphi \\ -\cos \varphi\end{array}\right)+2 l_{2} \dot{\varphi}_{2}^{2}\left(\begin{array}{c}\cos \varphi \\ \sin \varphi\end{array}\right)+2 l_{1} \ddot{\alpha}\left(\begin{array}{c}\cos \alpha \\ \sin \alpha\end{array}\right)-2 l_{1} \dot{\alpha}^{2}\left(\begin{array}{c}\sin \alpha \\ -\cos \alpha\end{array}\right)$

$\vec{r}_{C / B}=2 l_{1} \vec{e}_{r 2} ; \vec{v}_{C / B}=2 l_{1} \dot{\alpha} \vec{e}_{\varphi 2}$;

\section{Point F:}

$$
\begin{aligned}
& \vec{a}_{F}=2 l_{2} \ddot{\varphi}\left(\begin{array}{c}
\sin \varphi \\
-\cos \varphi
\end{array}\right)+2 l_{2} \dot{\varphi}_{2}^{2}\left(\begin{array}{c}
\cos \varphi \\
\sin \varphi
\end{array}\right)+l_{1} \ddot{\alpha}\left(\begin{array}{c}
\cos \alpha \\
\sin \alpha
\end{array}\right)-l_{1} \dot{\alpha}^{2}\left(\begin{array}{c}
\sin \alpha \\
-\cos \alpha
\end{array}\right) \\
& \vec{v}_{F / B}=l_{1} \dot{\alpha} \vec{e}_{\varphi 2} ; \vec{r}_{F / B}=l_{1} \vec{e}_{r 2}
\end{aligned}
$$

\section{Point K:}

$$
\begin{gathered}
\vec{a}_{K}=\left(\begin{array}{c}
\sin \alpha_{2} \\
-\cos \alpha_{2}
\end{array}\right)\left(\frac{\ddot{l}_{3}}{2}-\frac{l_{3}}{2} \dot{\alpha}_{2}^{2}\right)+\left(\begin{array}{c}
\cos \alpha_{2} \\
\sin \alpha_{2}
\end{array}\right)\left(\dot{l}_{3} \dot{\alpha}+\frac{l_{3}}{2} \ddot{\alpha}_{2}\right)+\left(\begin{array}{c}
\sin \alpha \\
-\cos \alpha
\end{array}\right) l_{2} \ddot{\varphi}_{2}+\left(\begin{array}{c}
\cos \varphi \\
\sin \varphi
\end{array}\right) l_{2}\left(\dot{\varphi}_{2}\right)^{2} \\
\vec{v}_{K / E}=\frac{\dot{l}_{3}}{2} \vec{e}_{r 3}+\frac{l_{3}}{2} \dot{\alpha}_{2} \vec{e}_{\varphi 3} ; \vec{r}_{K / E}=\frac{l_{3}}{2} \vec{e}_{r 3}
\end{gathered}
$$

Point Q:

$\vec{a}_{Q / C}=l_{5} \ddot{\alpha}_{3} \vec{e}_{\varphi 4}-l_{5} \dot{\alpha}_{3} 2 \vec{e}_{r 4} ; \vec{v}_{Q / C}=l_{5} \dot{\alpha}_{\varphi 4} ; \vec{r}_{Q / C}=l_{5} \vec{e}_{r 4}$

\section{Point $\mathrm{K}_{2}$ :}

$\vec{a}_{K_{2} / Q}=\vec{e}_{r 5}\left(\frac{\ddot{l}_{4}}{2}-\frac{l_{4}}{2} \dot{\alpha}_{3}^{2}\right)+\vec{e}_{\varphi 5}\left(\dot{l}_{4} \dot{\alpha}_{3}+\frac{l_{4}}{2} \ddot{\alpha}_{3}\right) ; \vec{v}_{K_{2} / Q}=\frac{\dot{l}_{4}}{2} \vec{e}_{r 5}+\frac{l_{4}}{2} \dot{\alpha}_{3} \vec{e}_{\varphi 5} ; \vec{r}_{K_{2} / Q}=$ $\frac{l_{4}}{2} \vec{e}_{r 5}(12)$

From these data, the motion function of each important point above could be calculated. In the next step, the motor torque for the proposed exoskeleton was found.

Based on Figure 5, the forces $\mathrm{Q}_{y}$ and $\mathrm{Q}_{x}$ are the solutions these equations below:

$$
\begin{aligned}
& \left\{\begin{array}{l}
Q_{y} l_{5} \cos \alpha_{6}+Q_{x} l_{5} \sin \alpha_{6}=M_{C}+P_{6} l_{5} \cos \alpha_{6}-\frac{4}{3} m_{6} l_{5}^{2} \\
-Q_{y} l_{4} \cos \alpha_{5}+Q_{x} l_{4} \sin \alpha_{5}=P_{5} \frac{l_{4}}{2} \cos \alpha_{5}+\frac{1}{3} m_{5} l_{4}^{2} \cdot \ddot{\alpha}_{4}
\end{array}\right. \\
& \Leftrightarrow\left\{\begin{array}{l}
Q_{y}=f_{1}\left(\alpha ; \varphi ; \alpha_{3} ; M_{C}\right) \\
Q_{x}=f_{2}\left(\alpha ; \varphi ; \alpha_{3} ; M_{C}\right)
\end{array}\right.
\end{aligned}
$$

Where

$$
\begin{aligned}
& Q_{x}=I_{2 x}+F_{\text {damper } 2 x} \\
& Q_{y}=I_{2 x}+F_{\text {damper } 2 y}
\end{aligned}
$$

When the leg is pulled up into the air, the values $M_{c}$ and $\alpha_{3}$ are equal to zero. Hence, it can be simplified to approximately these functions with respect only to variables $\alpha$ and $\varphi$. When the value of $\mathrm{Q}_{y}$ and $\mathrm{Q}_{x}$ are calculated, the value of the others and the essential magnitude of motor torque can be found.

From Figure 5, after some manipulation of mathematic modifications, the results of force were obtained as follows:

$$
\begin{aligned}
& C_{x}=-Q_{x}-m_{6} a_{Q x} ; C_{y}=P_{6}-Q_{y}+m_{6} a_{Q y} ; F_{x}=Q_{x}-m_{5} a_{K_{2} x} ; F_{y}= \\
& Q_{y}+P_{5}+m_{5} a_{K_{2 y}} \quad(16) \\
& A_{x}=m_{1} a_{F x}+m_{a} a_{Q x}+m_{5} a_{K_{2 x}}+m_{4} a_{K x}+\left(m_{2}+m_{3}\right) a_{E x} \quad(17) \\
& A_{y}=P_{1}+P_{2}+P_{3}+P_{4}+P_{5}+P_{6}+m_{4} a_{K y}+m_{5} a_{K_{2 y}}+m_{6} a_{Q y}+\left(m_{2}+m_{3}\right) a_{E y} \\
& \text { Where } A_{x} \text { and } A_{y} \text { are the forces generated by the individual. }
\end{aligned}
$$




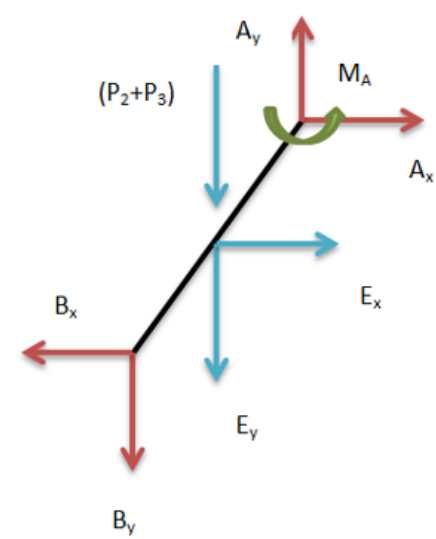

(a)

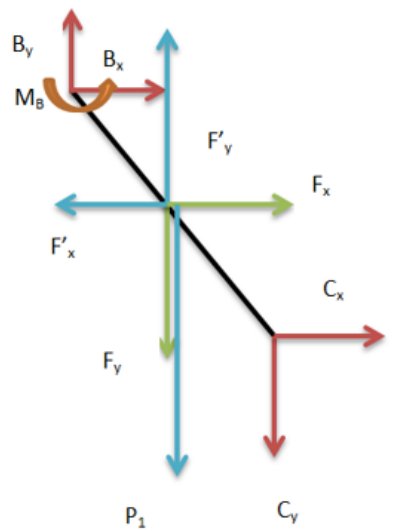

(b)

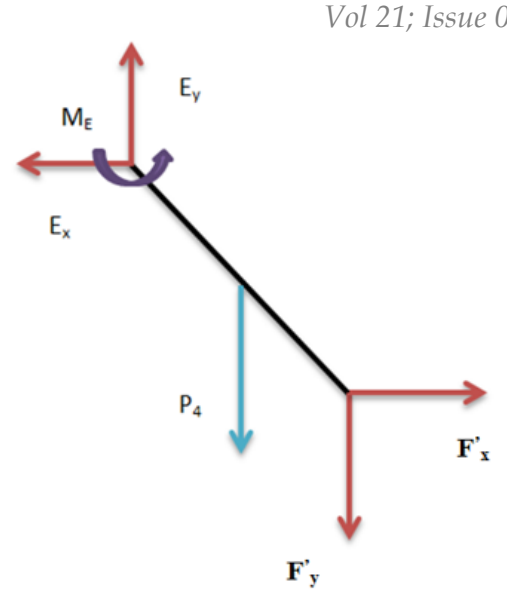

(c)

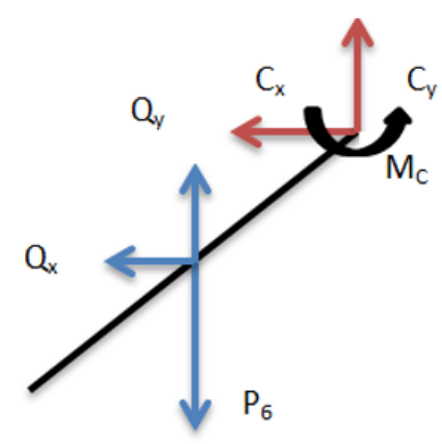

(d)

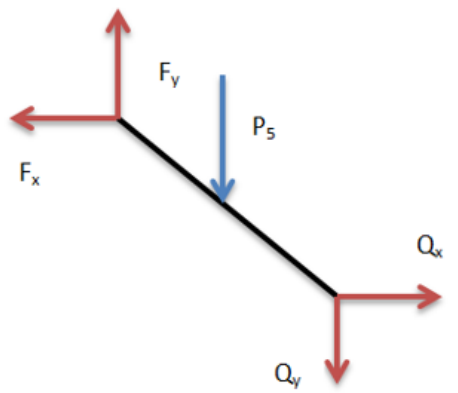

(e)

Figure 5. Force and Torques: (a) thigh, (b) first damper, (c) shank, (d) second damper, (e) foot (Note: $\mathbf{A}_{y}$, $\mathbf{A}_{x}, \mathbf{E}_{y}, \mathbf{E}_{x}, \mathbf{B}_{y}, \mathbf{B}_{x}, \mathbf{F}_{x}, \mathbf{F}_{y}, \mathbf{F}_{x}, \mathbf{F}_{y}, \mathbf{C}_{x}, \mathbf{C}_{y}, \mathbf{Q}_{x}, \mathbf{Q}_{y}, \mathbf{I}_{x}, \mathbf{I}_{y}, \mathbf{I}_{2 x}, \mathbf{I}_{2 y}$ areinternal forces).

The objective of this study was to design of an exoskeleton which can support people with difficulties pulling their legs up. The magnitude of torque at the knee must be optimized to zero. Hence, the results of the moments were found as follows:

$M_{B}=0 \Rightarrow F_{y}^{1} l_{1} \cos \gamma-F_{x}^{1} l_{1} \sin \gamma=\frac{4}{3} m_{1} \alpha l_{1}^{2}+F_{y} l_{1} \cos \gamma+P_{1} l_{1} \cos \gamma+2 C_{y} l_{1} \cos \gamma-$ $F_{x} l_{1} \sin \gamma-2 C_{x} l_{1} \sin \gamma$

$$
\begin{aligned}
& M_{A}=\frac{4}{3} m_{2} l_{2} \varphi_{2}+m_{3} l_{2}^{2} \varphi_{2}-l_{2} \cos \varphi\left(P_{2}+P_{3}+-F_{y}^{\prime}+P_{4}+m_{4} a_{K_{y}}+2 F_{y}+2 P_{1}+2 C_{y}+2 m_{1} a_{F_{y}}\right) \\
& -l_{2} \sin \varphi\left(-F_{x}-m_{4} a_{K_{x}}-2 m_{1} a_{K_{x}}+2 C_{x}+2 F_{x}\right)
\end{aligned}
$$

$M_{E}=\frac{1}{3} m_{4} l_{3}^{2} \alpha_{2}+P_{4} \frac{l_{3}}{2} \cos \theta+F_{y}^{\prime} l_{3} \cos \theta-F_{x}^{\prime} l_{3} \sin \theta$

The relation of torques and forces in the equilibrium state was expressed as follows:

$$
M_{A}+M_{E}-P_{4} x_{K / A}-P_{1} x_{F / A}-P_{5} x_{K_{2} / A}-P_{6} x_{Q / A}-\left(P_{2}+P_{3}\right) x_{E / A}=0
$$

The location $x$ can be determined due to the kinematic relations above. From equations (19-22), the values of $F^{\prime}{ }_{x}, ; F^{\prime}{ }_{y}$ could be solved, where:

$$
\begin{aligned}
& F_{x}^{\prime}=I_{x}+F_{\text {damper } 1 x} \\
& F_{y}^{1}=I_{y}+F_{\text {damper } 1 y}
\end{aligned}
$$


It was noted that the values of these forces are functions of 3 angles $\left(\alpha, \varphi, \alpha_{3}\right)$. However, the value of $\alpha_{3}$ in this state could be seen equal to 0 . Finally, from equation (21), it can be easily seen that $M_{E}$ could be calculated by $F_{x}{ }_{x}, F_{y}$ where:

$$
M_{E}=f_{3}(\alpha ; \varphi)
$$

\subsection{Proposed MR damper}

The piston of the proposed MR damper for exoskeleton is shown in Figure 6 and includes two parts: an electric coil and circular permanent magnets. Under normal conditions, the magnet already creates a magnetic field inside the MR damper. Due to that, the current supply for the damper is not necessary and, thus, energy saving is considerable. When the process needs to change the yield strength of damper, the current is supplied. The direction of current influences the magnetic field intensity created by the magnet. The intensity of magnet increases when vectors $\mathbf{B}$ of the current and magnet are in the same direction, and decreases when they are in opposite directions. This principle is a new approach to control the MR damper to increase the ready-state in large vibration control.

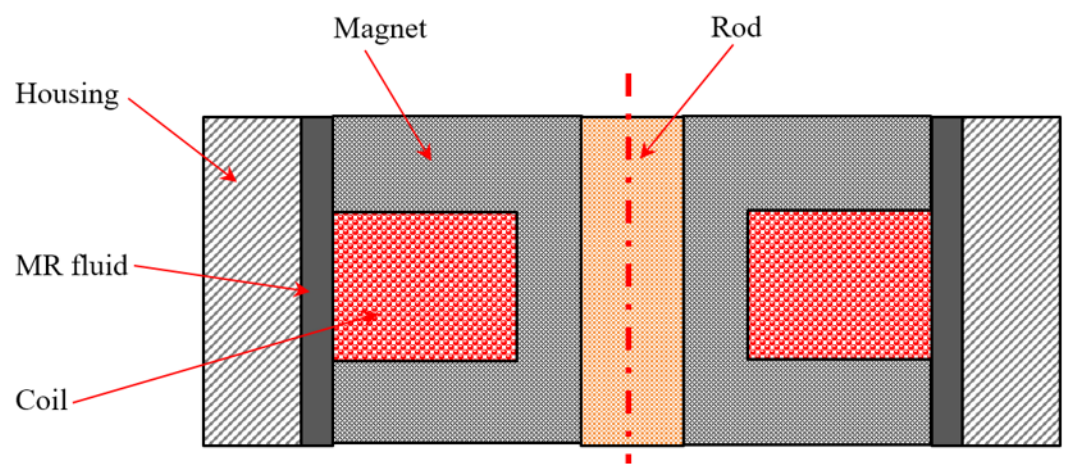

Figure 6. Configuration of MR damper.

The magnetic field phenomena are described through the ANSYS simulation for 3 situations, and captured in the images below:

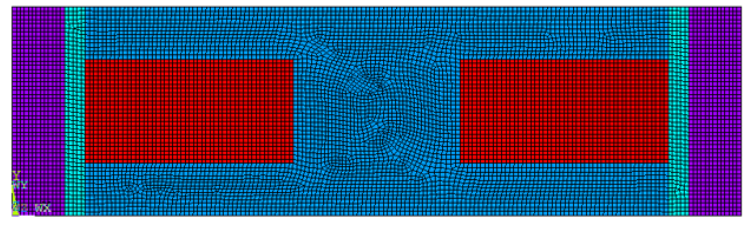

Figure 7. Meshing in the optimization of the proposed MR damper.

\section{Formulation and Optimization of the Proposed MR Damper}

Before optimization, the damping force of the two MR dampers must be defined. The dragging force (created by the MR damper) is calculated under the incompressible condition and the assumption that the yield stress always stays constant between two plates. Besides, the flow of MR fluid must be laminar and there is a neglecting of centrifugal force. The force of MR fluid inside the damper was calculated as follows:

$$
F_{M R}=\tau A=4 \pi \tau r t
$$




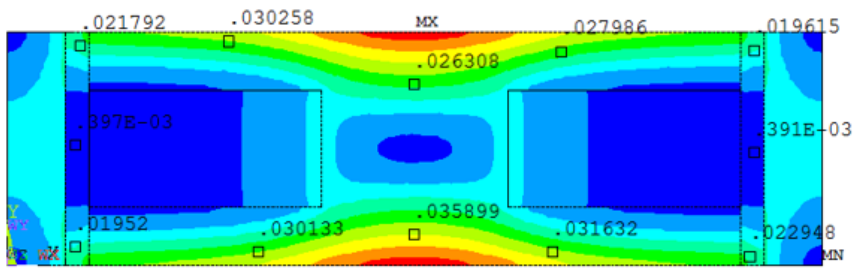

(a)

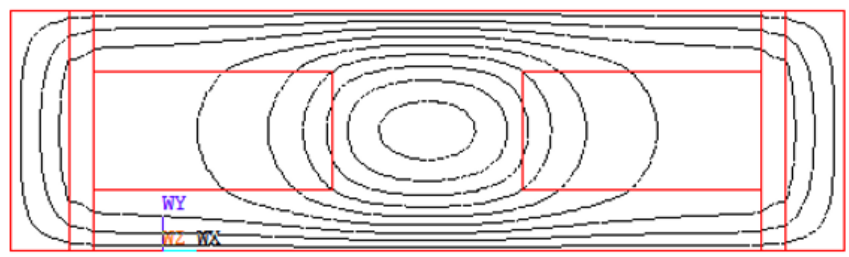

(b)

Figure 8. Only the magnet generates a magnetic field: (a) contour, (b) flux lines.

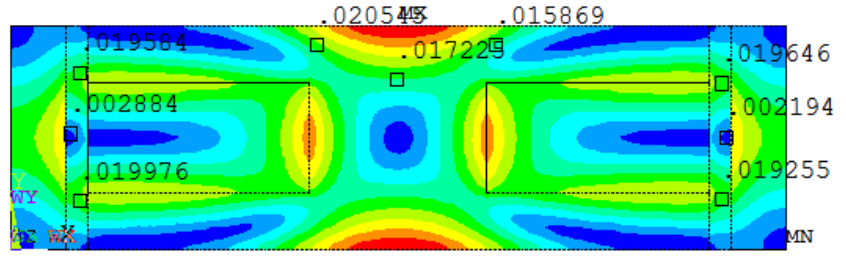

(a)

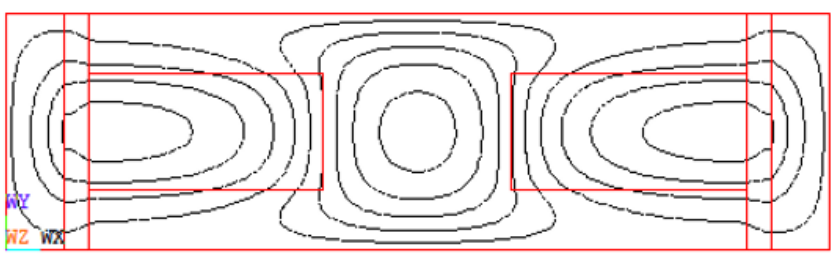

(b)

Figure 9. The magnet and coil generate a magnetic field with opposite vectors B when current is 2A: (a) contour, (b)flux lines.

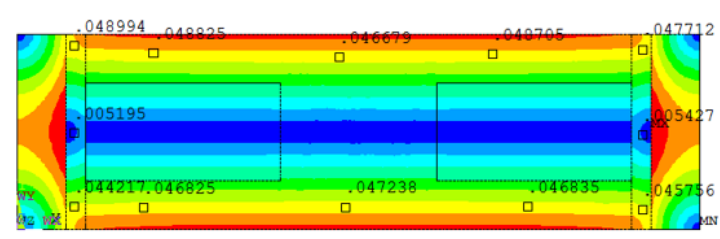

(a)

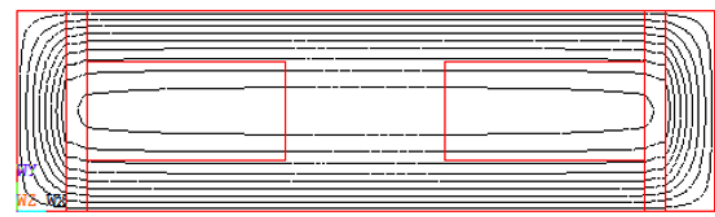

(b)

Figure 10. The magnet and coil generate a magnetic field with same side vectors B when current is 2A: (a) contour, (b) flux lines.

Hence, the total damping force of the MR damper was determined by Esteki et al. (2014)

$F_{\text {damper }}=\left(F_{M R}+2 f\right) \operatorname{sgn}(\stackrel{\square}{x})$

The relation between total shear stress and yield stress was determined by the Bingham model Wang et al. (2009)

$\tau=\left(\tau_{y}(H)+\eta|\square| \gamma \mid\right) \operatorname{sgn}(\stackrel{\square}{\gamma})$

In this model, the relation can be simplified to the equation below:

$\tau=\tau_{y}(H)+\eta \frac{x}{d} \quad(29)^{\tau=\tau_{y}(H)+\eta \frac{x}{d}}{ }^{(29)} \tau=\tau_{y}(H)+\eta \frac{x}{d} \quad(29)$

Due to practical experiments, the function $\tau_{y}(H)$ is related the form of Figure 11.

The function $\tau_{y}(H)$ can be expressed approximately by the least square curve fitting method Do et al. (2015):

$\tau_{y}(H)=C_{0}+C_{1} H+C_{2} H^{2}+C_{3} H^{3}$

In this damper, the general type MR fluid (MRF132-LD) was applied. Then, the value of coefficients $C_{0}, C_{1}, C_{2}, C_{3}$ were, respectively, approximate to: $-1.026,0.42,-0.00116$, and $1.0513(10)^{-6}$.

The magnetic field intensity through an electrical core was calculated by the Ampere's law: 
Table 2. Damper parameter

\begin{tabular}{|c|c|c|c|}
\hline $\begin{array}{l}\text { Sym- } \\
\text { bol }\end{array}$ & Definition & $\begin{array}{l}\text { Sym- } \\
\text { bol }\end{array}$ & Definition \\
\hline \multirow[t]{2}{*}{$F_{M R}$} & The force of MR fluid inside the damper & $\tau_{y}$ & Yield stress \\
\hline & Shear stress of the MR fluid & $g_{\gamma}$ & Shear rate \\
\hline$A$ & $\begin{array}{l}\text { The charged area of the damper (the surface area } \\
\text { of piston where flux density is thickest) }\end{array}$ & $N$ & Turns of coil \\
\hline$r$ & The Axial radius of the piston & $I$ & The applied current \\
\hline$t$ & $\begin{array}{l}\text { The thickness of magnetic pole (the line where flux } \\
\text { density is thickest) }\end{array}$ & $d$ & $\begin{array}{l}\text { The gap between housing and } \\
\text { piston (filled with MR fluid) }\end{array}$ \\
\hline$f$ & $\begin{array}{l}\text { Coulomb friction force between the piston and the } \\
\text { seals }\end{array}$ & $\eta$ & Viscosity of MR fluid \\
\hline$h$ & The height of coil section & $\mu$ & $\begin{array}{l}\text { Permeability of substance (MR } \\
\text { fluid) }\end{array}$ \\
\hline$w$ & The width of coil section & $L$ & Thickness of magnet \\
\hline $\mathrm{Br}$ & Remanence field & $x(t)$ & The position function \\
\hline$F_{\text {dampe }}$ & ${ }_{r}$ Damping force of damper $\mathrm{x}(\mathrm{x}=1,2)$ & $a(t)$ & The acceleration function \\
\hline$F_{\text {spring }}$ & Spring force & $F_{\text {load }}$ & The burdened load \\
\hline$H_{\text {magn }}$ & etMagnetic field strength of magnet & $H_{\text {coil }}$ & Magnetic field strength of coil \\
\hline$H$ & Total magnetic field strength & $d c$ & The diameter of wire \\
\hline$\Delta x$ & The displacement & $k$ & Spring stiffness \\
\hline$m$ & Mass & Fsup- & External supporting force \\
\hline & & port & From human \\
\hline
\end{tabular}

Note: the symbols in this section are not related to the previous ones

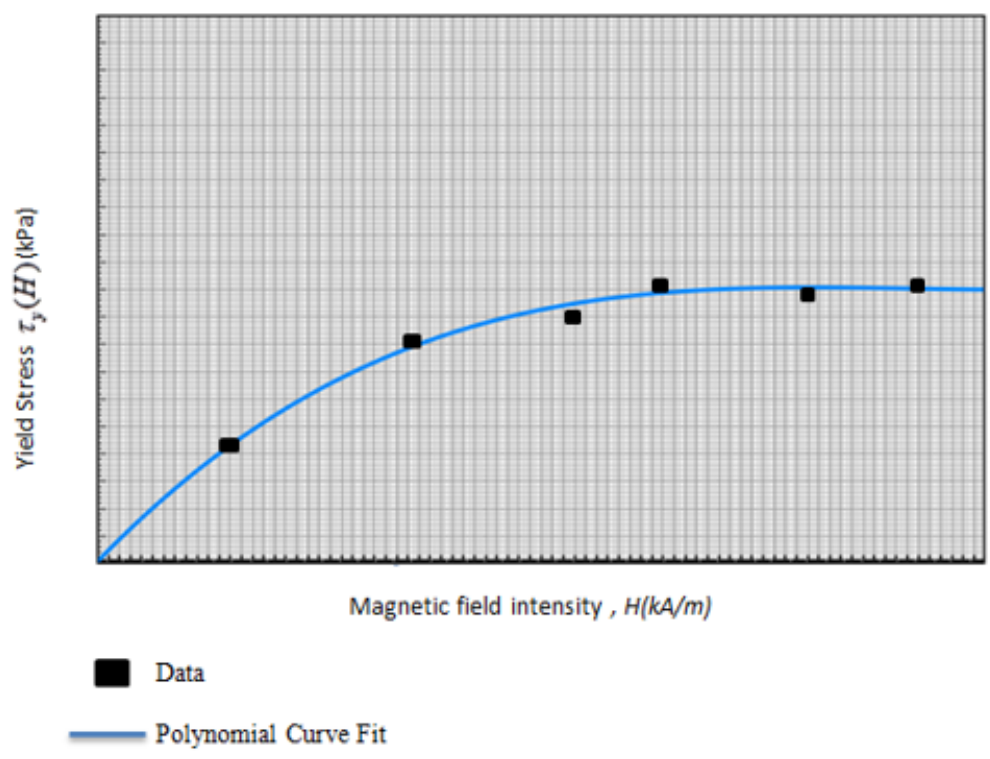

Figure 11. Yield stress versus magnetic field strength. 


$$
H_{\text {coli }}=N \frac{I}{2 d} \quad(31)
$$

In this shape of the damper, the number of turns in the coil could be calculated:

$N=\frac{4 w h}{\pi d_{c}^{2}}$

The magnetic field strength created by a magnet is very difficult to determine, in theory, because there are no simple formulas that calculate this field for the various magnetic shapes. Therefore, computer programs and modern devices are developed for this purpose. The magnetic flux density $\mathbf{B}$ of a magnet depends on the field which is independent of the magnet's geometry. In this model, it was calculated, approximately, based on the formula for block magnet flux density, though the shape of magnet in design was not a block.

$$
H_{\text {magnet }}=\frac{B_{r}}{\pi \mu}\left(\left(\arctan \left(\frac{L r}{2 d \sqrt{4 d^{2}+L^{2}+r^{2}}}\right)-\arctan \left(\frac{L r}{2(r+d) \sqrt{4(r+d)^{2}+L^{2}+r^{2}}}\right)\right)\right.
$$

The net magnetic field strength in this damper system was the result of the interaction between magnetic field strength of magnet and coil. This relation can be expressed by the formulation:

$H=H_{\text {magnet }}+H_{\text {coil }}$

From equations (29), (30), (31), the formulation of shear stress can be rewritten as follows:

$\tau=C_{0}+C_{1}\left(H_{\text {magnet }}+\frac{N I}{2 d}\right)+C_{2}\left(H_{\text {magnet }}+\frac{N I}{2 d}\right)^{2}+C_{3}\left(H_{\text {magnet }}+\frac{N I}{2 d}\right)^{3}+\frac{\eta}{d} \stackrel{\square}{x}$

From equation (26), the force was finally calculated to be:

$F_{M R}=4 \pi r t\left(C_{0}+C_{1}\left(H_{\text {magnet }}+\frac{N I}{2 d}\right)+C_{2}\left(H_{\text {magnet }}+\frac{N I}{2 d}\right)^{2}+C_{3}\left(H_{\text {magnet }}+\frac{N I}{2 d}\right)^{3}+\frac{\eta}{d} x\right)$

According to research at Southern Methodist University in Dallas, the characteristic of ground reaction force during time is depicted in the below two-mass model of the human body graph Figure 12:

\section{Two-Mass Model}

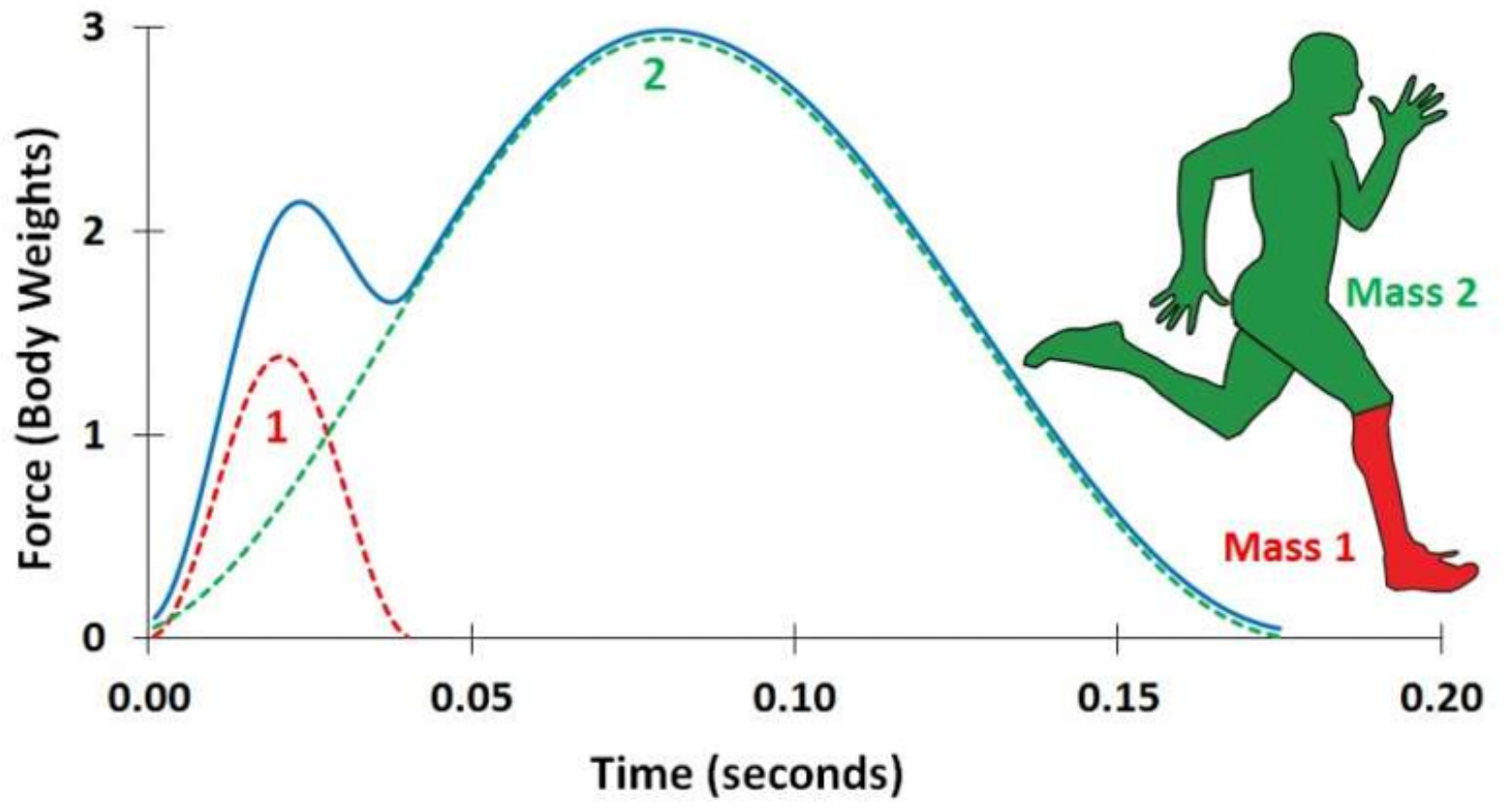

Figure 12. Dashed red curve: the reaction force of the ground with the lower limb; dashed blue curve: the force corresponding to the motion of the body'sremainder; solid blue curve: the net reaction ground force 224. 
When the foot lands on the ground, the body receives a burden generated from the weight and extra force during the motion. The combination of damper 2 and spring will play the main roles supporting this (the mass of damper and spring is neglected).

$$
\begin{aligned}
& F_{\text {support }}-F_{\text {load }}-F_{\text {damper } 2 y}-F_{\text {spring }}=0 \Leftrightarrow F_{\text {support }}-F_{\text {load }}-F_{\text {damper } 2 y}-k \Delta x=0 \Leftrightarrow \\
& F_{\text {support }}-F_{\text {load }}-F_{\text {damper } 2} \cos \theta-k \Delta x \cos \theta=0 \quad(37) \\
& \theta \text { is the angle formed by damping force and the horizontal line. }
\end{aligned}
$$

At a specific time, the cubic equation of $\mathrm{H}$, respective to acceleration and position function, was formed:

$$
\tau_{y}(H)=\frac{\frac{F_{\text {support }}-F_{\text {load }}-k \Delta x \cos \theta}{\operatorname{sgn}\left(\frac{\square}{x}\right)}-2 f}{4 \pi r t \cos \theta}-\eta \frac{\dot{x}}{d}
$$

In this part, the load force was equal to the net reaction ground force and the supporting force could be replaced totally by the damper and spring force $\left(F_{\text {support }}=0\right)$. When the motion goes upward, the damping force function is adjusted and optimized so that the motion stops at the highest suitable position but is still soft and comfortable. For the damper 1 , because it was not combined with the spring, $k \Delta x$ component will be neglected in equation (40). The load in this situation corresponds to the body remainder (does not contain the lower limbs contacting the ground).

An optimized product is the most compacted one which also can supply the largest needed force. In this study, the objective damping force of the second damper was $250 \mathrm{~N}$. After optimization, the value of damping force was $248.67 \mathrm{~N}$ with the yield stress as $201.831 \mathrm{kPa}$. Similarly, the objective damping force of the first damper was $150 \mathrm{~N}$. The value obtained after optimization was $150.27 \mathrm{~N}$ with the yield stress of $752.85 \mathrm{kPa}$. It should be noted that the maximum applied current was $2 \mathrm{~A}$ for both dampers. The results of optimization are shown in Figures 8,9 and 10. In these figure, the magnetic flux density of the two dampers is in range of the boundaries. Hence, the performance of these dampers is guaranteed and can be manufactured in the future.

\section{Conclusion}

In this study, a new exoskeleton with two MR dampers is presented. Its forces and torques were analyzed to find the final values for design of the MR dampers. In the proposed damper, a new direction of control was suggested. The control follows the variation of the vector of magnetic lines, which was created by an electric coil and a block of permanent magnet. Based on the force and torque analysis, the section of damper can be designed and optimized. The results of optimization show that the requirement of design can be feasibly obtained, and that the magnetic flux density is in range of limitation of all materials. In the future, the proposed exoskeleton with MR dampers will be manufactured and tested.

\section{Acknowledgements}

This research is funded by Vietnam National Foundation for Science and Technology Development (NAFOSTED) under grant number 107.01-2017.28.

\section{References}

Southern methodist university, "new study connects running motion to ground force, provides patterns for any runner", january 31, 2017. 
Choi, S.-B., Li, W., Yu, M., Du, H., Fu, J., and Do, P. X. (2016). State of the art of control schemes for smart systems featuring magneto-rheological materials. Smart Materials and Structures, 25(4):043001.

I. Sahin, T. Engine, S. Cesmeci (2010). Comparison of some existing parametric models for magnetorheological fluid dampers. Smart materials and structures, 19(3):035012.

Do, X. P. and Choi, S.-B. (2015). High loaded mounts for vibration control using magnetorheological fluids: review of design configuration. Shock and Vibration, 2015.

Do, X. P., Shah, K., and Choi, S.-B. (2015). Damping force tracking control of mr damper system using a new direct adaptive fuzzy controller. Shock and Vibration, 2015.

Esteki, K., Bagchi, A., and Sedaghati, R. (2014). Dynamic analysis of electro-and magneto-rheological fluid dampers using duct flow models. Smart Materials and Structures, 23(3):035016.

McLaughlin, G., Hu, W., and Wereley, N. M. (2014). Advanced magnetorheological damper with a spiral channel bypass valve. Journal of Applied Physics, 115(17):17B532.

Phu, D. X., An, J.-H., Choi, S.-B., et al. (2017a). A novel adaptive pid controller with application to vibration control of a semi-active vehicle seat suspension. Applied Sciences, 7(10):1055.

Phu, D. X., Choi, S. B., Lee, Y. S., and Han, M. S. (2014a). Design of a new engine mount for vertical and horizontal vibration control using magnetorheological fluid. Smart Materials and Structures, 23(11):117001.

Phu, D. X., Choi, S. B., Lee, Y. S., and Han, M. S. (2014b). Design of a new engine mount for vertical and horizontal vibration control using magnetorheological fluid. Smart Materials and Structures, 23(11):117001.

Phu, D. X., Choi, S.-M., and Choi, S.-B. (2017b). A new adaptive hybrid controller for vibration control of a vehicle seat suspension featuring mr damper. Journal of Vibration and Control, 23(20):33923413.

Phu, D. X., Hung, N. Q., and Choi, S.-B. (2017c). A novel adaptive controller featuring inversely fuzzified values with application to vibration control of magneto-rheological seat suspension system. Journal of Vibration and Control, page 1077546317740479.

Wang, D. H., Ai, H. X., and Liao, W. H. (2009). A magnetorheological valve with both annular and radial fluid flow resistance gaps. Smart materials and structures, 18(11):115001. 